\title{
Robust Adaptive Control of a Flexible Transmission System Using Multiple Models
}

\author{
Alireza Karimi and Ioan Doré Landau
}

\begin{abstract}
An application of the multiple models adaptive control based on switching and tuning to a flexible transmission system will be presented. This approach has been considered in order to assure high control performance in the presence of large load variation on the system. The advantages of the multiple models adaptive control system with respect to the classical adaptive control will be illustrated via the experimental results. It will also be shown that the robustness of the adaptive control system can be improved with the appropriate shaping of a sensitivity function. The use of a recently developed parameter estimation algorithm based on the minimization of the closed-loop output error in the multiple models scheme will also improve the performance of the system in the tuning phase and will make the adaptation algorithm insensitive to unmodeled output disturbances.
\end{abstract}

Index Terms-Adaptive control, closed-loop identification, flexible structures, multimodel control, robustness, switching.

\section{INTRODUCTION}

$\mathbf{F}$ LEXIBLE systems with very low damping factor are in general very difficult to control in the presence of large load variation. However, a high-performance controller is normally needed for this type of systems, particularly in aerospace applications. The experiments have shown that a fixed high-performance controller designed for one loading may lead to instability for another loading. The robust stability may be achieved only by a low-performance robust controller.

In this paper, we consider a flexible transmission system with two very oscillatory vibration modes subject to large load variations (leading to about $100 \%$ variation of the first vibration mode). This system has been the subject of a benchmark on robust digital control at the European Control Conference in Rome 1995 [1]. Several robust control design methods have been considered and examined on the real system, including $H_{\infty}$ control [2]-[4], QFT [5], [6], CRONE [7], GPC [8], pole placement with sensitivity function shaping [9], [10] and direct minimization of a performance with a model free approach [11]. The robust stability of the system has been assured by all of the robust controller proposed, but a very high-performance fixed parameter controller for all of the loadings could not be achieved.

Adaptive control is a potential solution for improving the performances of this system for large parameter variations. An adaptive control approach for this system has already been considered by M'Saad and Hejda in [12] using the generalized pre-

Manuscript received March 31, 1998. Recommended by Associate Editor, F. Ghorbel.

A. Karimi is with the Electrical Engineering Department of Sharif University of Technology, Tehran, Iran.

I. D. Landau, is with the Laboratoire d'Automatique de Grenoble (CNRSINPG-UJF); Ensieg, BP. 46, 38402 St. Martin d'Hères, France.

Publisher Item Identifier S 1063-6536(00)01784-X dictive control combined with a robust least squares parameter estimator. However, the classical robust adaptive control approach leaves unsolved two important problems.

1) Transient responses caused by abrupt and large changes in the load cannot be handled by the adaptive system (the adaptive system is not enough fast to follow the parameter variations and unacceptable transients occur).

2) In the absence of an excitation signal, the parameter drift caused by the unmodeled output disturbances can render the system unstable.

Different techniques have been proposed in the literature to solve the problem of parameter drift, like using a dead-zone in the parameter adaptation algorithm or using a permanent excitation signal added to the reference signal of the system. Although these techniques assure the stability of the system, they deteriorate the performances. Another technique, so called adaptation freezing, which is normally used in this situation is difficult to tune and works only with a particular reference input and a particular type of disturbances.

The novelty of this paper is the presentation of an adaptive control scheme for the flexible transmission system which removes the disadvantages of the classical robust adaptive control. This scheme is based on the following algorithms.

1) Use of multiple models approach to adaptive control [13].

2) Replacement of the least squares estimator by a recently developed "closed-loop output error" parameter estimation scheme [14].

The use of an adaptive controller based on multiple models and switching will allow the transient responses to be improved in the presence of large and fast parametric variations [15]-[17]. In this approach, we suppose that a set of models for different operating points is a priori known (which is the case for the flexible transmission, since the system can be identified for a certain numbers of loads). Then at every instant a controller corresponding to the model yielding the minimum of a performance index is used to compute the control input. The precision of the control can be further improved using an adaptive model (a model whose parameters are updated with a parameter adaptation algorithm) in the set of models. This method together with a stability analysis was proposed by Narendra and Balakrishnan in [13].

To solve the parameter drift problem, we will use a closed-loop adaptation error algorithm which is based on the use of the closed-loop output error between the output of the true closed-loop system and of a predictor of the closed-loop reparameterized in terms of the controller and of an estimated plant model. The "closed-loop output error" algorithm (CLOE), is by its structure insensitive to output disturbances 


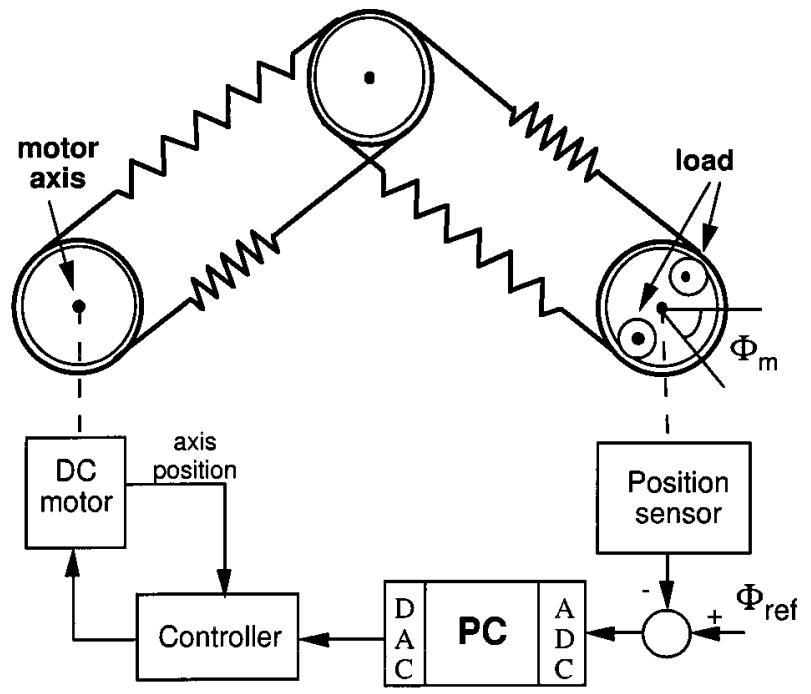

Fig. 1. Schematic diagram of the flexible transmission.

and allows the need for adaptation freezing in the absence of external excitation to be removed. Furthermore, this algorithm is dedicated to plant model identification in closed loop which assures asymptotically unbiased parameter estimates when the plant model is in the model set. In the presence of unmodeled dynamics the bias frequency distribution of the parameters in this algorithm is weighted by two closed-loop sensitivity functions which makes the identified model very suitable for robust control design.

The rest of this paper is organized as follows. The flexible transmission is described in Section II. The principles of the multiple models adaptive control based on switching and tuning will be presented in Section III. Section IV explains the basis of the CLOE parameter estimation algorithm. A robust controller using the pole placement combined with sensitivity function shaping method is considered as the underlying linear control design for the adaptive control law and it is presented in Section V. The experimental results are given in Section VI and finally, Section VII presents the concluding remarks.

\section{SYSTEM DESCRIPTION}

The flexible transmission system built at Laboratoire d'Automatique de Grenoble (INPG-CNRS), France, consists of three horizontal pulleys connected by two elastic belts. The schematic diagram and the photo of the system are shown, respectively, in Figs. 1 and 2. The first pulley is driven by a de motor whose position is controlled by local feedback. Since the dynamic of this feedback loop is much faster than that of the mechanical parts, it can be neglected in the analysis of the system. The objective is to control the position of the third pulley which may be loaded with small disks. The system input is the reference for the axis position of the first pulley. A PC is used to control the system. The sampling frequency is $20 \mathrm{~Hz}$.

The system is characterized by two low damped vibration modes (with damping factors of less than 0.05), subject to a large variation in the presence of load. Fig. 3 gives the amplitude of the frequency characteristics of the identified discrete-time models for three different loadings; no-load, half-load $(1.8 \mathrm{Kg})$

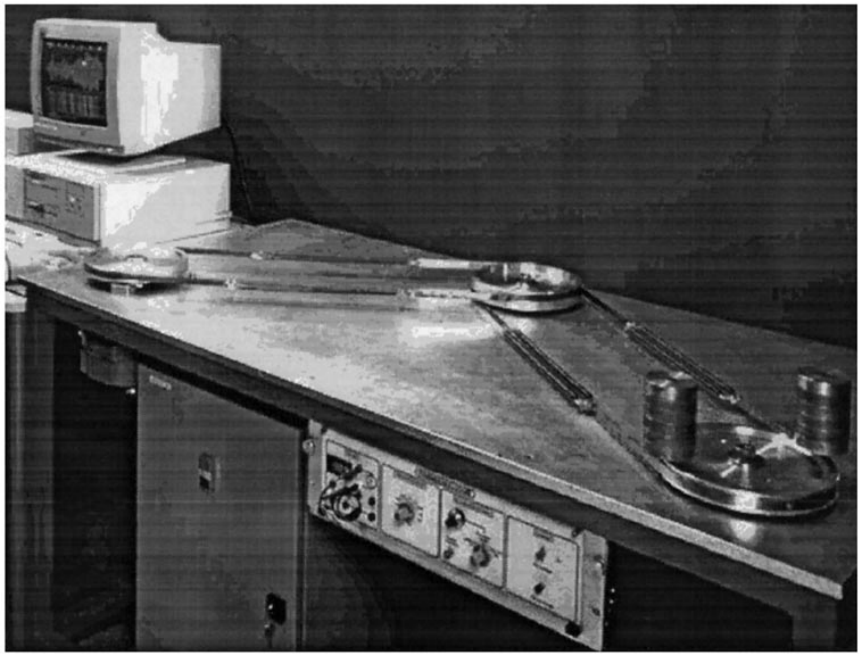

Fig. 2. View of the flexible transmission system.

and full-load $(3.6 \mathrm{Kg})$. The frequencies are normalized by the sampling frequency $f_{s}$. A variation of about $100 \%$ of the frequency of the first vibration mode occurs when passing from the full loaded case to the unloaded case. The system has a pure time delay equal to two sampling periods and an unstable zero. The discrete-time plant is described by the following transfer operator:

$$
H\left(q^{-1}\right)=\frac{q^{-d} B\left(q^{-1}\right)}{A\left(q^{-1}\right)}
$$

where $q^{-1}$ is the backward shift operator, $d$ is the plant pure time delay and

$$
\begin{aligned}
& B\left(q^{-1}\right)=b_{1} q^{-1}+\cdots+b_{n_{B}} q^{-n_{B}} \\
& A\left(q^{-1}\right)=1+a_{1} q^{-1}+\cdots+a_{n_{A}} q^{-n_{A}} .
\end{aligned}
$$

The parameters of the three identified models are given below.

No load:

$$
\begin{aligned}
B\left(q^{-1}\right)= & 0.41156 q^{-1}+0.52397 q^{-2} \quad d=2 \\
A\left(q^{-1}\right)= & 1-1.35277 q^{-1}+1.55021 q^{-2}-1.27978 q^{-3} \\
& +0.91147 q^{-4} .
\end{aligned}
$$

Half load:

$$
\begin{aligned}
B\left(q^{-1}\right)= & 0.16586 q^{-1}+0.18133 q^{-2} \quad d=2 \\
A\left(q^{-1}\right)= & 1-1.92005 q^{-1}+2.1151 q^{-2}-1.81622 q^{-3} \\
& +0.91956 q^{-4} .
\end{aligned}
$$

Full load:

$$
\begin{aligned}
B\left(q^{-1}\right)= & 0.09553 q^{-1}+0.11116 q^{-2} \quad d=2 \\
A\left(q^{-1}\right)= & 1-2.05182 q^{-1}+2.23744 q^{-2}-1.91294 q^{-3} \\
& +0.90643 q^{-4} .
\end{aligned}
$$




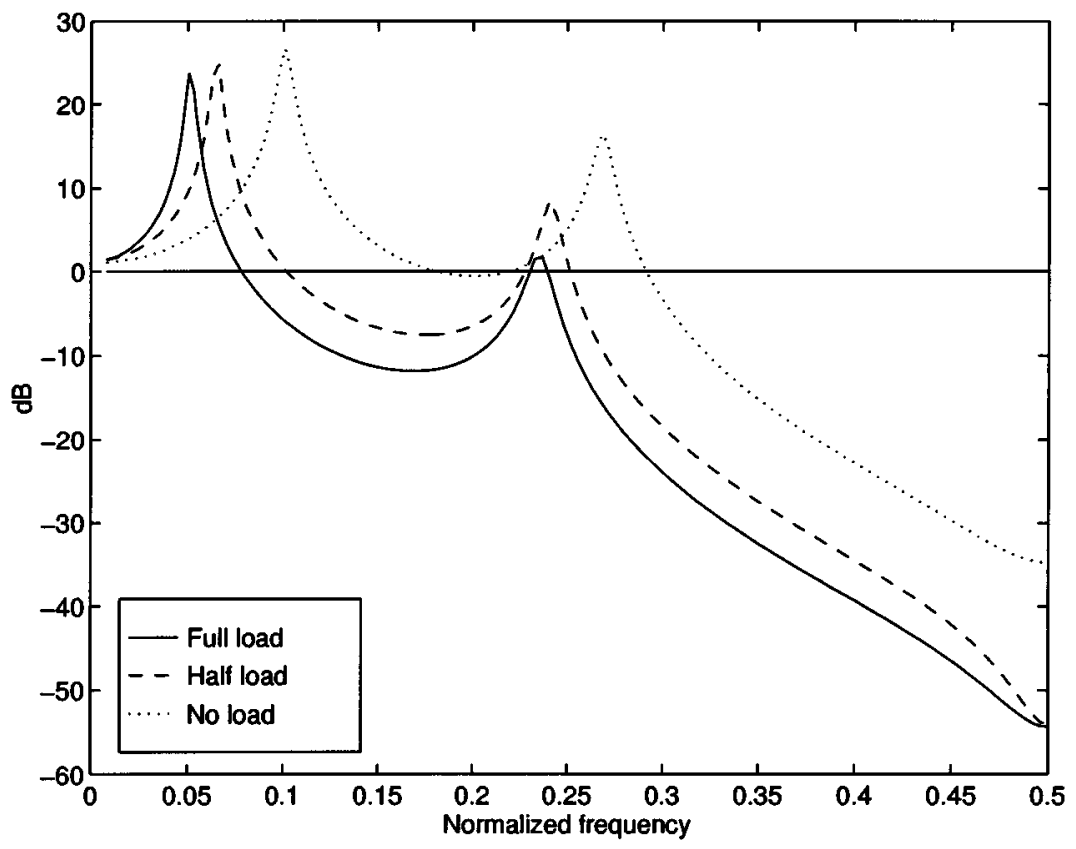

Fig. 3. Frequency characteristics of the flexible transmission for various loads.

\section{Principles of AdAPtive Control With Switching AND TUNING}

The main idea of this method is to choose the best model for the plant from an a priori known set of models at every instant and apply the output of the corresponding controller to the plant. Since the number of available models is finite but the number of possible models is generally infinite, the identification is performed in two steps.

- The model with smallest error with respect to a criterion is rapidly chosen (switching).

- The parameters of the model are adjusted using a parameter adaptation algorithm (tuning).

The block diagram of this method is presented in Fig. 4. The input and output of the plant are $u(t)$ and $y(t)$, respectively. The control system contains $n$ models $G_{1}, \cdots, G_{n}$ which are either fixed or adaptive models. The identification error is defined as the difference between the output $\hat{y}_{i}$ of the model $G_{i}$ and the plant output

$$
\epsilon_{i}=y(t)-\hat{y}_{i}(t)
$$

For each model $G_{i}$, there is a controller $K_{i}$ that satisfies the control objective for $G_{i}$ (instead of $n$ controller we may have a parameterized controller $K\left(G_{i}\right)$ ). The performance criterion $J(t)$ which is used as the switching rule may be defined as follows [13]:

$$
\begin{gathered}
J_{i}(t)=\alpha \epsilon_{i}^{2}(t)+\beta \sum_{j=0}^{t} e^{-\lambda(t-j)} \epsilon_{i}^{2}(j), \\
\alpha \geq 0 \quad \beta, \lambda>0
\end{gathered}
$$

where $j$ is the time index and $\alpha$ and $\beta$ are the weighting factors on the instantaneous measures and the long-term accuracy. $\lambda$ is

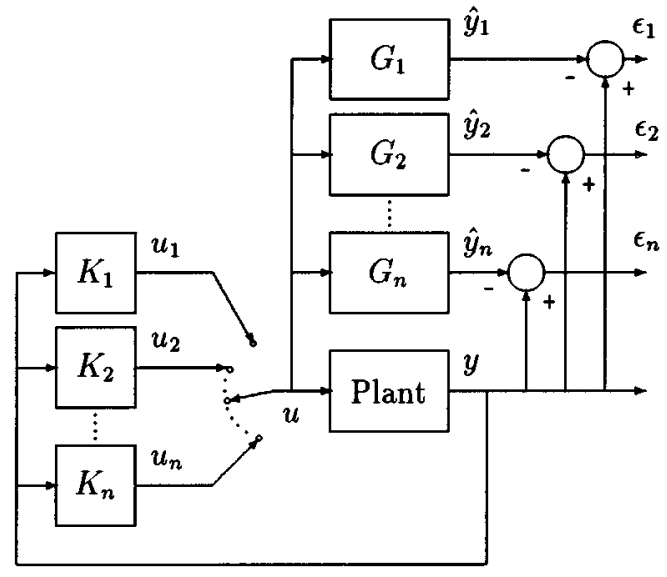

Fig. 4. Schematic diagram of the multiple models approach.

a forgetting factor which also assures the boundedness of the criterion for bounded $\epsilon_{i}(t)$. Then the design parameters for the switching part of the control system are $\alpha, \beta$ and $\lambda$. If we choose a large value for $\alpha / \beta$ and $\lambda$, we will obtain a very quick response to the abrupt parameter changing but a bad response with respect to disturbances. It means that, an output disturbance will generate an unwanted switching to another controller which result in a poor control. Contrary, a small value for $\alpha / \beta$ and $\lambda$ makes the criterion a good indicator of steady-state identifier accuracy which reduces the number of unwanted switching but leads to a slow response with respect to the parameter variation.

Another design parameter $T_{d}$, the minimum time delay between two switchings, plays an important role on the stability analysis and the performances of the system. A small value for $T_{d}$ gives too frequent switching and a large $T_{d}$ leads to a slow response system.

A hysteresis cycle with a design parameter $\gamma$ also may be considered between two switchings. It means that a switching 
to another controller will occur if the performance index concerning a model is improved by $\gamma$. A combination of two techniques (time delay and hysteresis) may also be considered.

The models in this approach may be either fixed or adaptive models. The parameters of the adaptive models may be initialized with the parameters of the last chosen fixed model in order to improve the adaptation speed. The stability analysis for several combinations of the models (all adaptive models, all fixed models, fixed models and one adaptive model, fixed models with one free-running and one reinitialized adaptive model) was given in [13]. In this paper, for the flexible transmission system, we consider the last case which is the most performant with the difference that the reinitialized adaptive model uses a new plant parameter estimation algorithm (CLOE) [14].

\section{Closed-LOOP OUtPut ERROR AdAPTATION AlgORITHM (CLOE)}

The closed-loop output error recursive adaptation algorithm presented in [14] is based on a reparameterized adjustable predictor for the closed-loop system in terms of a known fixed controller $K$ and an adjustable plant model $\hat{G}$. Fig. 5 shows the block diagram which is often used in closed-loop identification.

The output of the plant is described by

$$
y(t)=G u(t)+p(t)
$$

and the output of the predictor by

$$
\hat{y}(t)=\hat{G} \hat{u}(t) .
$$

It can be shown that the parameter estimates $\theta^{*}$ given by this algorithm satisfy asymptotically this relation [18]

$$
\begin{aligned}
\theta^{*} & =\arg \min _{\theta} \int_{-\pi}^{\pi}\left|S_{y p}\right|^{2}\left[|G-\hat{G}|^{2}\left|\hat{S}_{u p}\right|^{2} \phi_{r}(\omega)\right. \\
\left.+\phi_{p}(\omega)\right] d \omega &
\end{aligned}
$$

where $\theta$ is the vector of the parameter, $S_{y p}$ is the output sensitivity function for the real closed loop defined by

$$
S_{y p}=\frac{1}{1+K G}
$$

and $\hat{S}_{u p}$ is the input sensitivity function of the designed loop

$$
\hat{S}_{u p}=\frac{-K}{1+K \hat{G}}
$$

$\phi_{r}(\omega)$ and $\phi_{p}(\omega)$ are the spectra of reference and disturbance signals, respectively.

Equation (3) shows that first, the parameter estimates are affected only by the spectrum of the reference signal $\phi_{r}(\omega)$ and the noise spectrum $\phi_{p}(\omega)$ has asymptoticaly no effect on the minimum of the criterion with respect to $\theta$. Second, the difference between real plant $G$ and estimated one $\hat{G}$ is weighted by two sensitivity functions. It means that in the presence of unmodeled dynamics, we have more precision in the frequency zones where the modules of the sensitivity functions are large. Therefore the estimated model will be more accurate where the control system cannot tolerate large uncertainties. It can be also shown that this particular weighting is exactly what is required

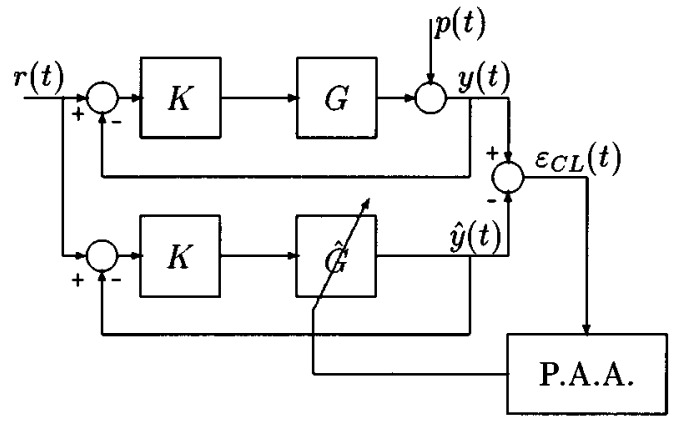

Fig. 5. Closed-loop output error identification scheme.

for minimizing a robust performance criterion in an iterative approach [18].

A recursive algorithm minimizing $\varepsilon_{C L}$ in Fig. 5 can be summarized as follows [14]. Consider the output of the system which can be described by

$$
y(t)=\frac{q^{-d} B\left(q^{-1}\right)}{A\left(q^{-1}\right)} u(t)+p(t)
$$

where $p(t)$ is the noise, assumed to be zero-mean with finite variance and independent with respect to the external excitation. Therefore

$$
y(t+1)=\theta^{T} \varphi(t)+A p(t+1)
$$

where

$$
\theta^{T}=\left[a_{1} \cdots a_{n_{A}}, b_{1} \cdots b_{n_{B}}\right]
$$

$$
\begin{aligned}
\varphi^{T}(t)= & {\left[-y(t) \cdots,-y\left(t-n_{A}+1\right),\right.} \\
& \left.u(t-d) \cdots, u\left(t-n_{B}+1-d\right)\right]
\end{aligned}
$$

$$
u(t)=K\left(q^{-1}\right)[r(t)-y(t)] .
$$

The a priori and a posteriori predictors for the plant output are, respectively, defined by

$$
\hat{y}^{\circ}(t+1)=\hat{\theta}^{T}(t) \phi(t)
$$

$$
\hat{y}(t+1)=\hat{\theta}^{T}(t+1) \phi(t)
$$

where

$$
\begin{aligned}
\hat{\theta}^{T}(t)= & {\left[\hat{a}_{1}(t) \cdots \hat{a}_{n_{A}}(t), \hat{b}_{1}(t) \cdots \hat{b}_{n_{B}}(t)\right] } \\
\phi^{T}(t)= & {\left[-\hat{y}(t) \cdots-\hat{y}\left(t-n_{A}+1\right)\right.} \\
& \left.\hat{u}(t-d) \cdots \hat{u}\left(t-n_{B}+1-d\right)\right] \\
\hat{u}(t)= & K\left(q^{-1}\right)[r(t)-\hat{y}(t)] .
\end{aligned}
$$



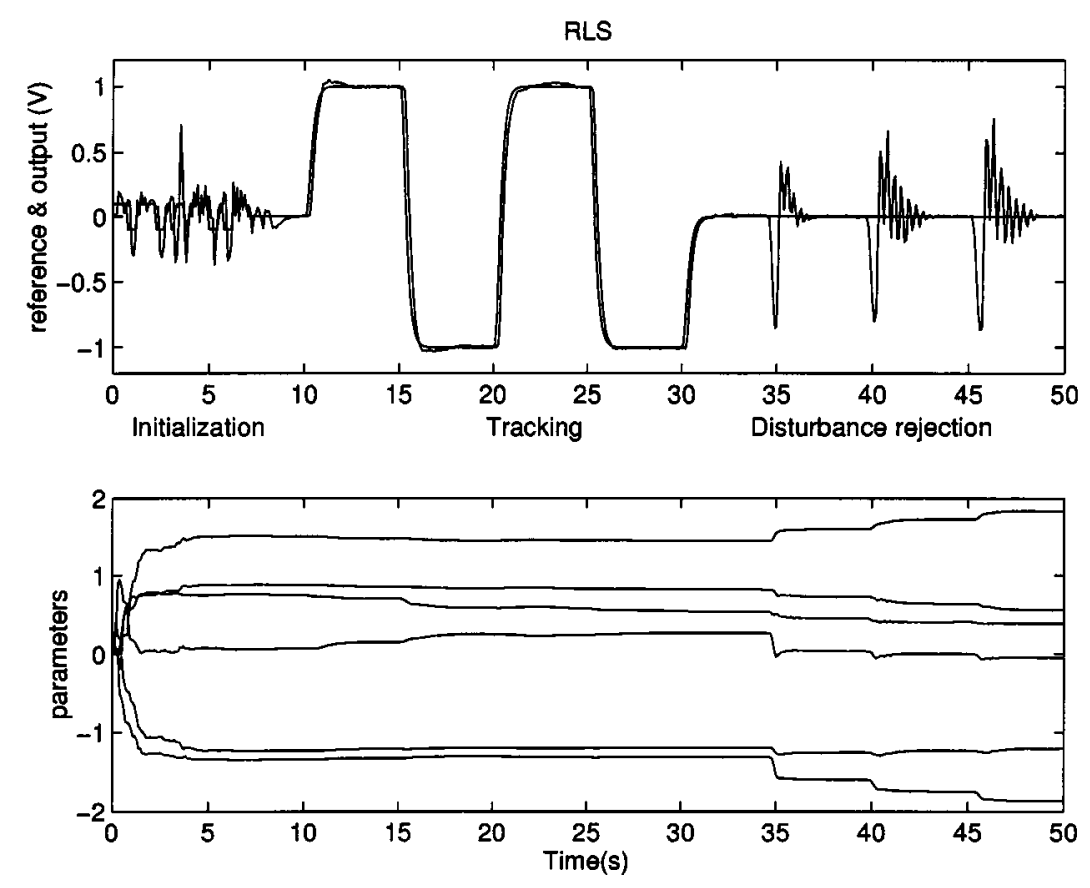

Fig. 6. Effects of output disturbances on the parameters in classical adaptive control.

Then the parameter adaptation algorithm has the form

$$
\begin{gathered}
\hat{\theta}(t+1)=\hat{\theta}(t)+F(t) \phi(t) \varepsilon_{C L}(t+1) \\
F^{-1}(t+1)=\lambda_{1}(t) F^{-1}(t)+\lambda_{2}(t) \phi(t) \phi^{T}(t) \\
0<\lambda_{1}(t) \leq 1, \quad 0 \leq \lambda_{2}(t)<2 \\
\varepsilon_{C L}(t+1)=\frac{y(t+1)-\hat{y}^{\circ}(t+1)}{1+\phi^{T}(t) F(t) \phi(t)} .
\end{gathered}
$$

The stability and the convergence analysis of this algorithm have been given in [14]. According to the analysis the real positivity of a closed-loop transfer function $(S / P$, where $S$ is the denominator of the controller and $P$ is the characteristic polynomial of the closed loop) plays an important role on the stability and the convergence of the algorithm. However, this real positivity condition may be weakened using a filtered regression vector defined as

$$
\phi_{f}(t)=\frac{S}{\hat{P}} \phi(t)
$$

where

$$
\hat{P}=\hat{A} S+q^{-d} \hat{B} R
$$

is an estimation of the closed-loop polynomial based on an estimation of $A$ and $B$. One uses the parameter adaptation algorithm of (16)-(19) in which $\phi(t)$ is replaced by $\phi_{f}(t)$. This algorithm named F-CLOE [14] will be used in the real-time experiments in Section VI because in this case the transfer function $\hat{P} / P$ should be positive real which is much milder than the real positivity condition on $S / P$. In these experiments $\hat{P}$ is in fact the desired closed-loop polynomial used for the pole placement method.

From (16), one can observe that the parameter estimates are not influenced by the output disturbances in the absence of the excitation (reference) signal, because in this algorithm the regression vector $\phi(t)$ depends only on the reference signal $r(t)$ which freezes automatically the drift of parameters. This can be clearly observed in the following real-time experiment.

Consider the flexible transmission system in the no-load case controlled by an adaptive pole placement controller. The system parameters are initialized in open loop when the plant is excited with a PRBS. Then the system will operate in closed loop (from 10s) and a square wave signal filtered by a reference model will be used as the closed-loop reference signal till $t=30 \mathrm{~s}$ and then is set to zero. Disturbances are applied after $t=34 \mathrm{~s}$. Fig. 6 shows the reference, the plant input and output and the evolution of the parameters using a classical least squares equation error adaptation algorithm (without adaptation freezing). We can see that a disturbance on the plant output causes a parameter drift which may render the system unstable. Fig. 7 shows the same experiment but using a closed-loop output error adaptation algorithm (the schematic diagram of this method is compared with the classical adaptive control scheme in Fig. 8). It can be observed that the output disturbances do not affect the parameters evolution. This property together with the asymptotic unbiasedness of the parameter estimates (in the absence of unmodeled dynamics) and the particular bias distribution of the identified model (in the presence of unmodeled dynamics) encouraged us to use this adaptation algorithm in an adaptive model in the multiple models scheme.

A preliminary study [19] shows that the existence of an excitation signal is necessary for the stability of an adaptive control system using the CLOE algorithm in a certain situation (when the closed-loop system becomes unstable in the absence of external excitation). However, this algorithm can be used in the 

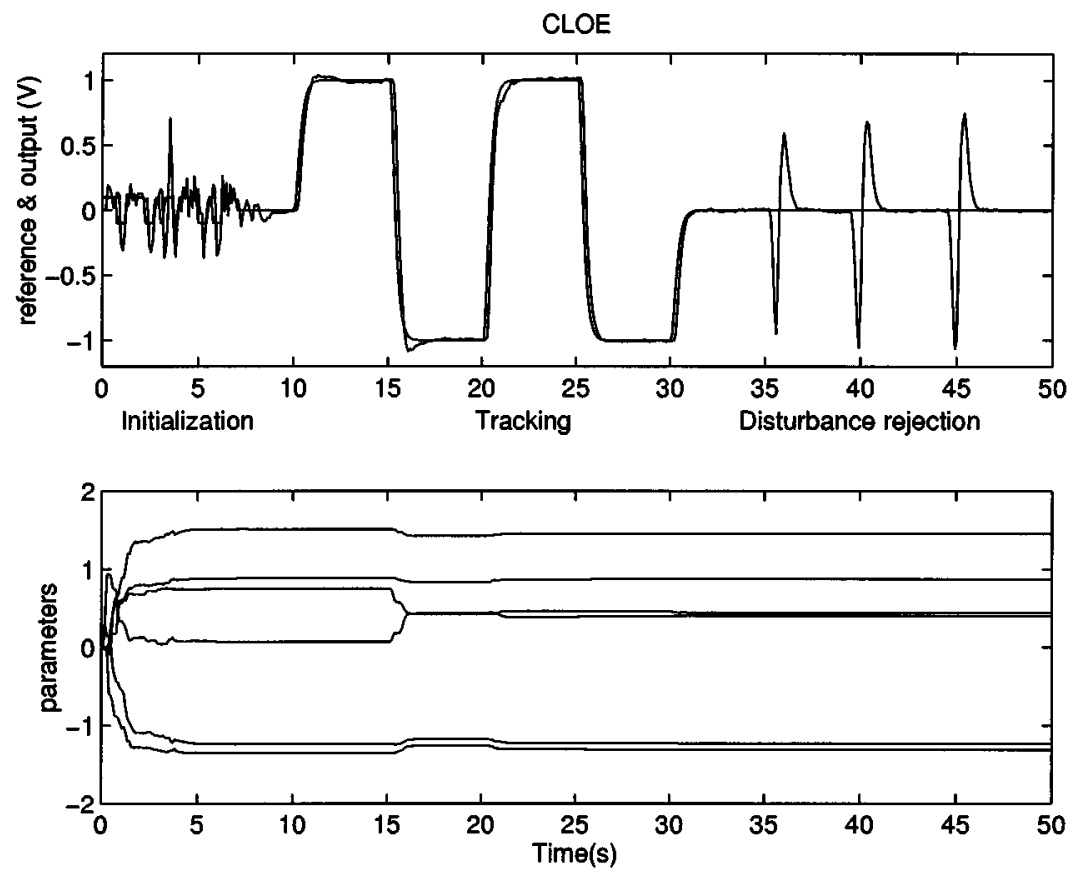

Fig. 7. Effects of output disturbances on the parameters in CLOE adaptive control.

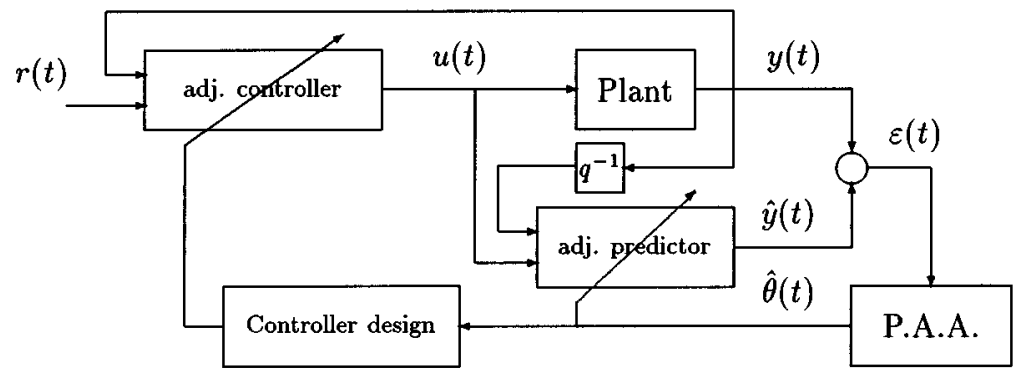

(a)

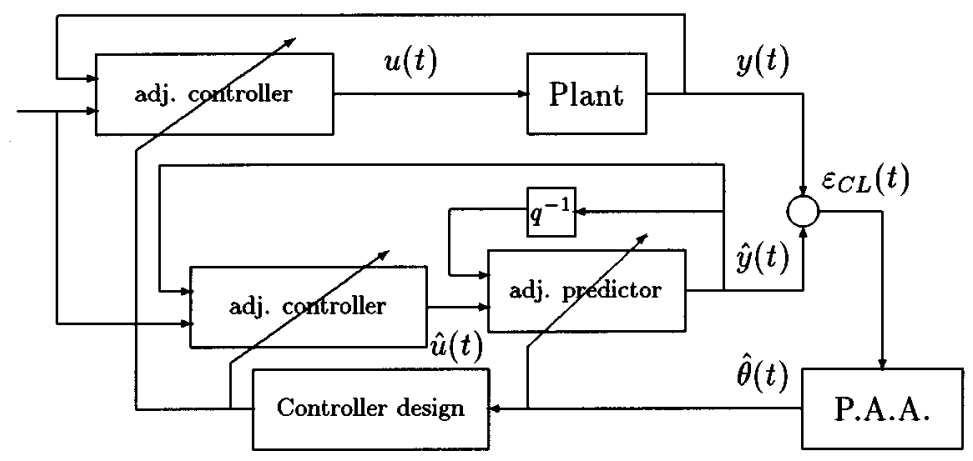

(b)

Fig. 8. Indirect adaptive control (a) classical adaptive control; (b) CLOE adaptive control.

multiple models scheme even if the stability condition for the algorithm is not satisfied. The reason is that in the absence of the excitation signal the parameters of the CLOE predictor remain unchanged and the adaptive model becomes a fixed model among the other fixed models, for which a stability analysis has been already presented [13].

\section{Robust CONTROL Design}

An adaptive control system responds directly to the structured parametric uncertainty by the adjustment of model parameters. Therefore, the unstructured modeling errors should be considered by the linear control law associated to the adaptive scheme. 


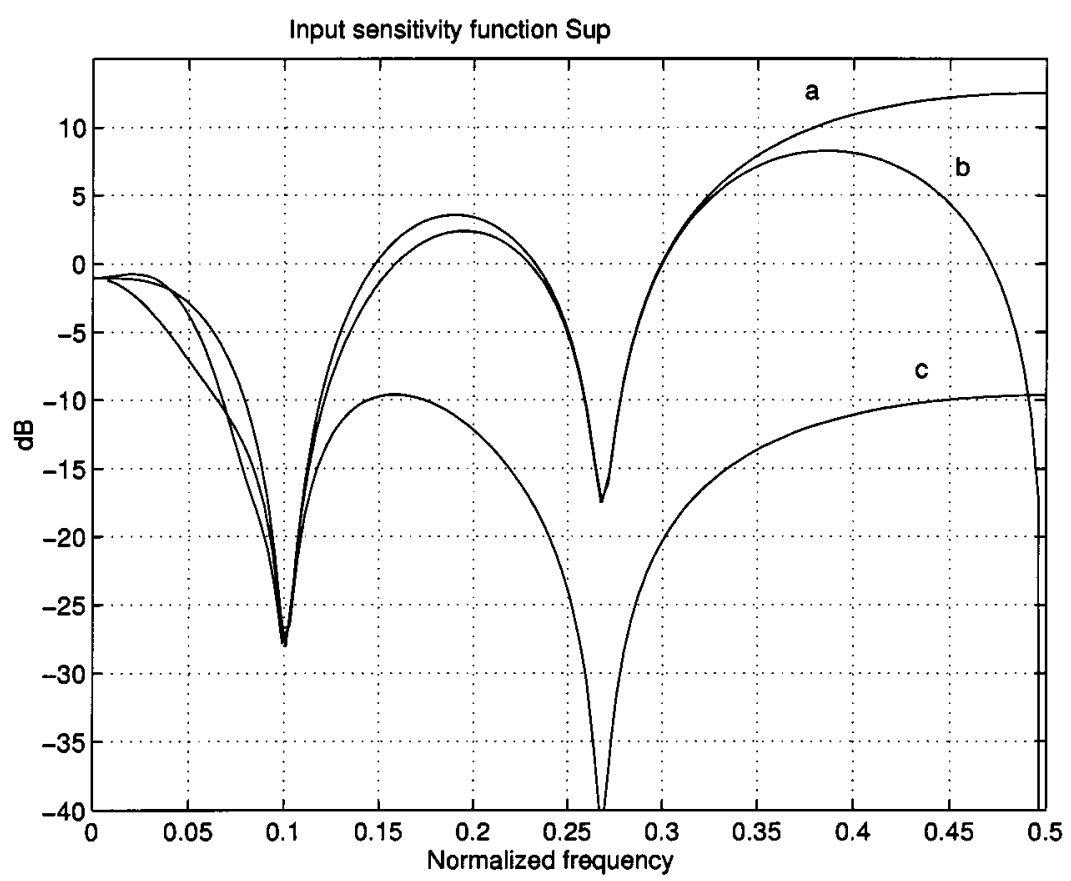

Fig. 9. Input sensitivity function shaping of the flexible transmission.

Since the unstructured modeling errors are in general large at high frequencies, the input sensitivity function $S_{u p}$ (the transfer function between output disturbance and plant input) which is related to additive uncertainties, should be low in these frequencies. For the flexible transmission system, this can be achieved using the pole placement with sensitivity function shaping [10] in the following ways.

1) Choice of a fixed term in the numerator of the controller in the form of $\left(1+\alpha z^{-1}\right)^{n}, 0.5 \leq \alpha \leq 1, n \geq 1$.

2) Choice of the auxiliary closed-loop poles near to the highfrequency poles of the plant.

The first one has an effect only in the very high frequencies (between 0.4 to $0.5 \mathrm{f} / f_{s}$ ), whereas the second one decrease significantly the input sensitivity function in middle and high frequencies (between 0.2 to $0.5 f / f_{s}$ ). The effects of these techniques can be easily shown in the following example.

Consider the discrete time model of the flexible transmission system in the no-load case. A first pole placement controller is designed with the following specifications.

1) A pair of complex dominant poles with the frequency of the first vibration mode of the plant model but with a damping factor of 0.8 .

2) Four auxiliary poles at 0.1 i.e., $\left(1-0.1 z^{-1}\right)^{4}$.

3) An integrator in the controller.

Fig. 9(a) shows the amplitude of the input sensitivity function $S_{u p}$ with a maximum of $12 \mathrm{~dB}$ at high frequency which signifies a bad robustness with respect to the additive uncertainties. The curve $b$ shows the effect of a fixed term $\left(1+z^{-1}\right)$ in the controller numerator. One observes that $S_{\text {up }}$ decreases in very high frequencies. But a pair of complex poles in the place corresponding to the second vibration mode of the plant will decrease significantly $S_{u p}$ in a very large band (curve $c$ ). Thus, in order to obtain a robust controller with respect to the mod- eling errors beyond the closed-loop band pass, auxiliary poles should be chosen near to the plant high-frequency poles. It is advisable to open the loop at $0.5 f / f_{s}$ and to remove from zero the remaining closed-loop poles which can be assigned.

\section{EXPERIMENTAL RESULTS}

In this section several real-time experiments will be carried out in order to show the performances of multiple models adaptive control in comparison with a classical one for the flexible transmission system. Different functions have been developed in order to realize the multiple models adaptive control on the VisSim [20] software environment.

Three discrete-time identified models for the no-load, halfload, and full-load cases together with an adaptive model with reinitialization using the CLOE algorithm are considered in the set of models (the adaptive free running model which is theoretically needed for deriving a stability proof was not necessary in the applications because there exists always a fixed model which leads to a stabilizing controller for all of the loadings). The bloc diagram of the control architecture is illustrated in Fig. 10. $G_{1}, G_{2}$ and $G_{3}$ represent unloaded, half loaded, and full loaded models, respectively. $\hat{G}$ is the adaptive model using the CLOE algorithm. At every instant, the supervisor chooses the best model $G^{*}$ according to the performance index of (5) and the control input $u(t)$ is determined based on this model and using the pole placement method. The following specifications are considered for the pole placement control design.

Dominant Poles: A pair of complex poles with a frequency of $6 \mathrm{rad} / \mathrm{s}\left(0.048 \mathrm{f} / f_{s}\right)$ and a damping factor of 0.9 . This frequency corresponds to the natural frequency of the first vibration mode of the full loaded model.

Auxiliary Poles: A pair of auxiliary poles with a frequency of $33 \mathrm{rad} / \mathrm{s}\left(0.263 \mathrm{f} / f_{s}\right)$ and a damping factor of 0.3 which 


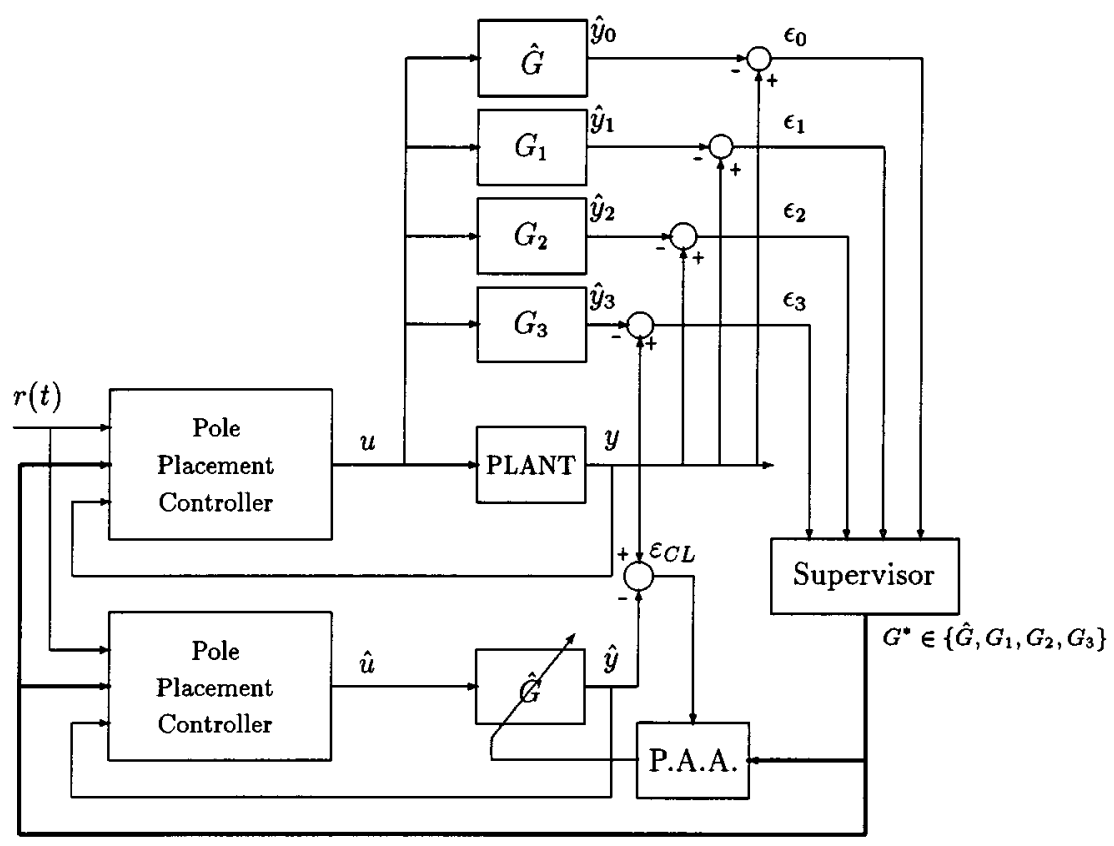

Fig. 10. Bloc diagram of the real time experiments.

is close to the high-frequency poles of open-loop models and the real poles $\left(1-0.5 z^{-1}\right)^{3}\left(1-0.1 z^{-1}\right)^{4}$. Fixed Terms: The controller contains an integrator and a term $\left(1+z^{-1}\right)^{2}$ in the numerator to reduce the input sensitivity function in very high frequencies.

Reference Model (Tracking): A second-order system with $\omega_{0}=6 \mathrm{rad} / \mathrm{s}$ and a damping factor of 1 is chosen as the reference model for tracking.

An estimation of the control input $\hat{u}(t)$ is also computed which will be used to determine $\hat{y}(t)$ and $\varepsilon_{C L}(t)$ in the CLOE algorithm.

A two degree of freedom RST controller for the pole placement is considered, i.e.,

$$
S\left(q^{-1}\right) u(t)=-R\left(q^{-1}\right) y(t)+T\left(q^{-1}\right) y^{*}(t)
$$

where $y^{*}(t)$ is the reference trajectory stored in the computer or generated via the reference model.

The aim and objective of the three real time experiments are summarized as follows:

Experiment 1: Comparision of fixed robust control versus adaptive multimodel control which shows a reduction of tracking error for multimodel adaptive system.

Experiment 2: Comparison of classical adaptive control versus adaptive multimodel control which results in a faster parameter adaptation and better reliability for multimodel adaptive system.

Experiment 3: Studying the behavior of the adaptive multimodel control system when the fixed models $(0 \%, 50 \%$, and $100 \%$ load) do not correspond to reality $(25 \%, 75 \%$ load) which illustrates fast parameter adaptation.

\section{A. Experiment 1}

A square wave signal with an amplitude of $1 \mathrm{~V}$ and a period of $10 \mathrm{~s}$ is applied on the input of the reference model and a disturbance is applied at the output of the plant (the angular position of the third pulley is changed slowly by hand and is released rapidly). The experiment is started without load on the third pulley and at the instants 9 s, 19 s, 29 s, and 39 s $25 \%$ of the total load is added on the third pulley. Therefore the system without load becomes full loaded in four stages. The parameters of the adaptive model are initialized to zero. The results of this experiment are compared with a fixed parameters robust controller which satisfies the specifications of the flexible transmission benchmark [1] in Fig. 11. Fig. 11 shows on one hand that very good performances in tracking and disturbance rejection are achieved using adaptive control with multiple models and on the other hand it shows that adaptive schemes proposed provides better performances than a good robust controller.

\section{B. Experiment 2}

The same control system with the same synthesis parameters as well as the same reference signal is again considered (without disturbance). The plant is initially full loaded and it passes to unloaded case in two stages (at $19 \mathrm{~s}$ and $29 \mathrm{~s}$ ). The results illustrated in Fig. 12 show the good tracking performances of the system. The switching diagram shows the best model chosen at every instant. In Fig. 12(d) 0 corresponds to the adaptive model and 1, 2, and 3 correspond to the no-load, half-load, and full-load models. The design parameters for the switching rule are: $\alpha=1, \beta=1$, and $\lambda=0.1$ and the performance index is computed for 100 samples (from $t-100 T_{s}$ up to $t$, where $T_{s}$ is the sampling period). These parameters lead to a rather fast control system with respect to the plant parameters variation.

It should be noticed that this experiment cannot be carried out using the classical adaptive controllers, because the control system generates signals greater than the value that can be tolerated by the real system. The maximum variation of angular position of the first and third pulleys is $90^{\circ}$ (corresponds to 2 V) from the equilibrium point. A proximity switch is utilized 

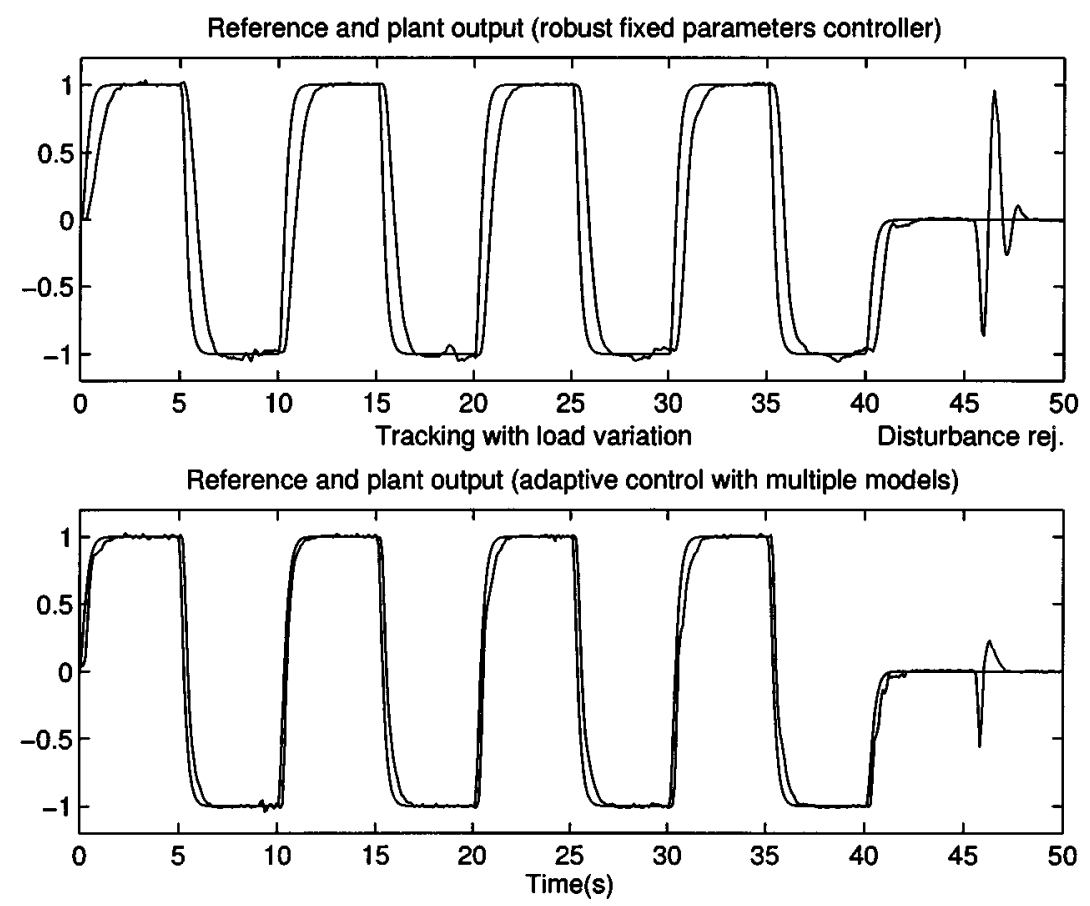

Fig. 11. Comparison of a fixed robust controller with an adaptive multimodel controller $(0 \% \rightarrow 100 \%$ in four stages at $9 \mathrm{~s}, 19 \mathrm{~s}, 29 \mathrm{~s}$ and $39 \mathrm{~s})$.
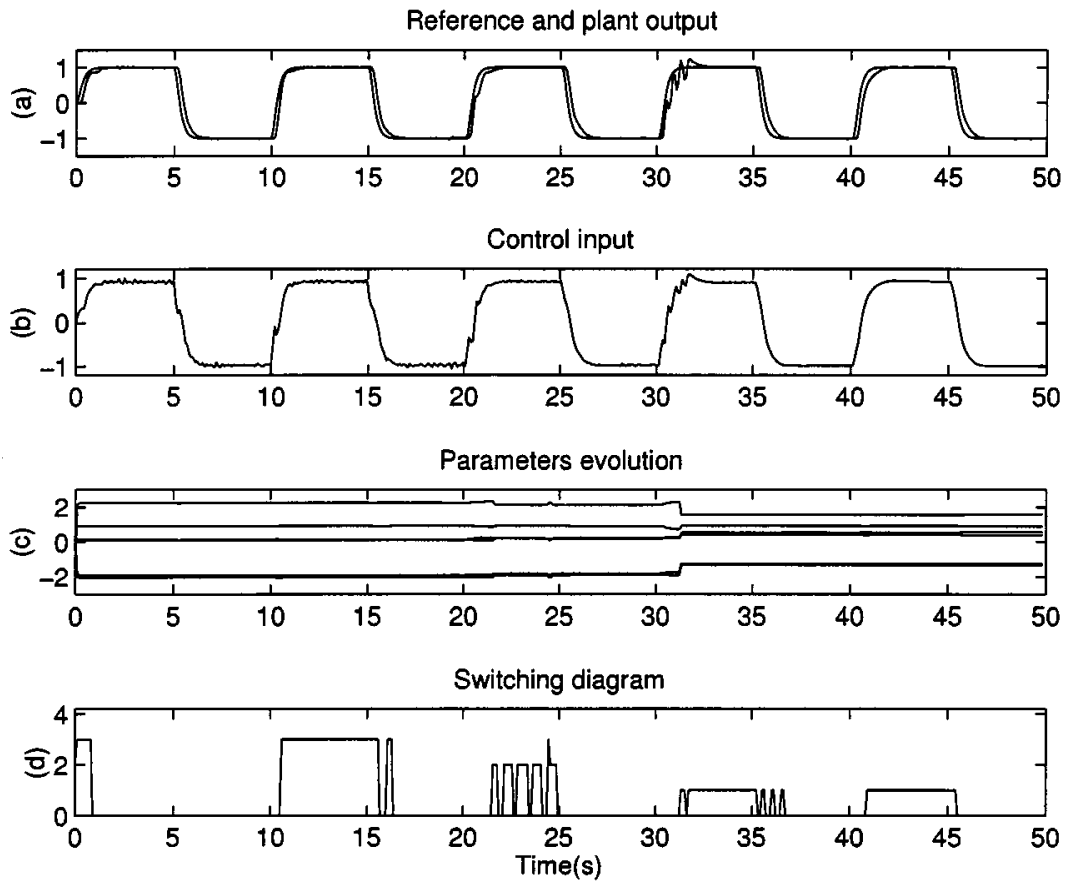

Fig. 12. Results of experiment $2(100 \% \rightarrow 0 \%$ in two stages at $19 \mathrm{~s}$ and $29 \mathrm{~s})$.

to shut down the system when the third pully rotates more than $90^{\circ}$. Fig. 13 shows the simulation results using a classical adaptive controller. The parameters of the model are initialized in open loop using a PRBS (pseudorandom binary sequence) and the loop is closed at $9 \mathrm{~s}$. The loads on the third pulley are taken off in two stages (at $19 \mathrm{~s}$ and $29 \mathrm{~s}$ ). In the first stage (at $19 \mathrm{~s}$ ) only a small deterioration in the response occures because the full-loaded and half-loaded models are not very different and the controller designed for the first one also stabilizes the second one. The parameter adaptation is not fast at this stage because the adaptation error is small. In the second stage, the controller for half-load does not stabilize the system for no-load. This generates large values of the adaptation error leading quickly to a stabilizing controller. Unfortunately the simulated output of the plant passes above $2 \mathrm{~V}$ during the adaptation transient and this is not acceptable on the real system. 

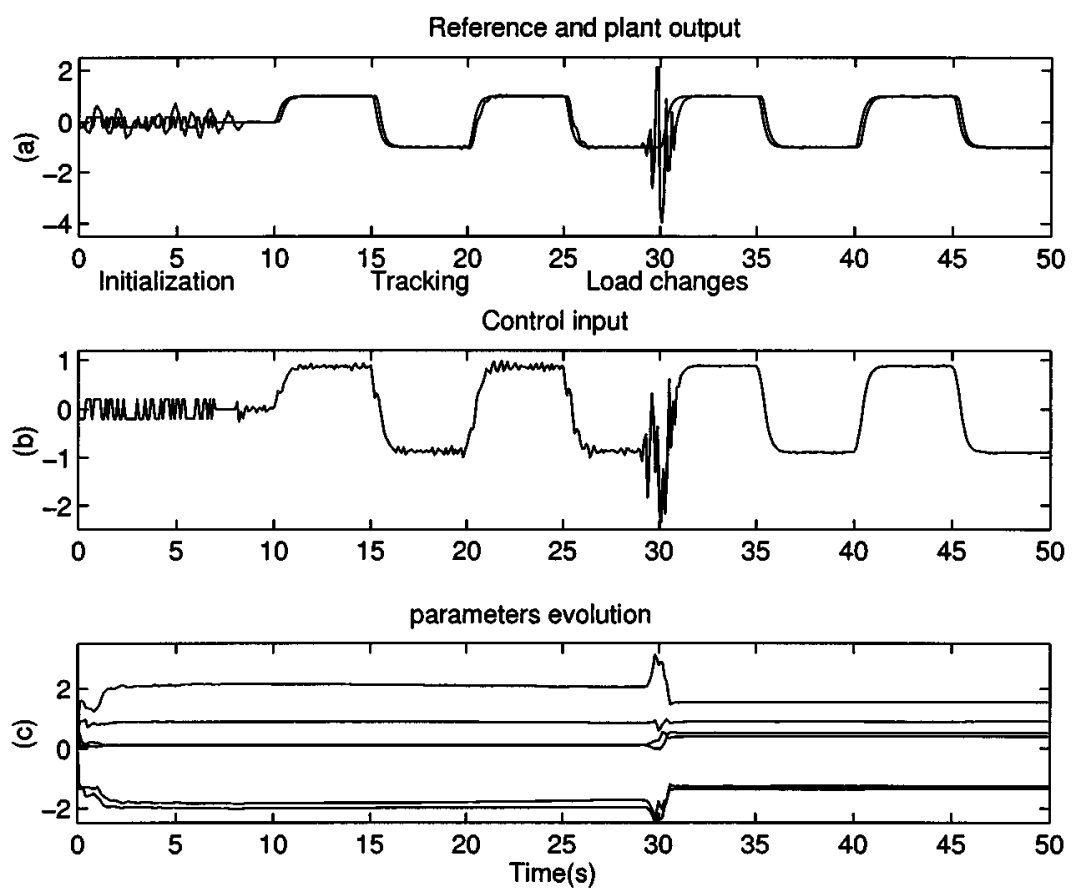

Fig. 13. Simulation results using classical adaptive control (100\% $\rightarrow 0 \%$ in two stages at $19 \mathrm{~s}$ and $29 \mathrm{~s})$.
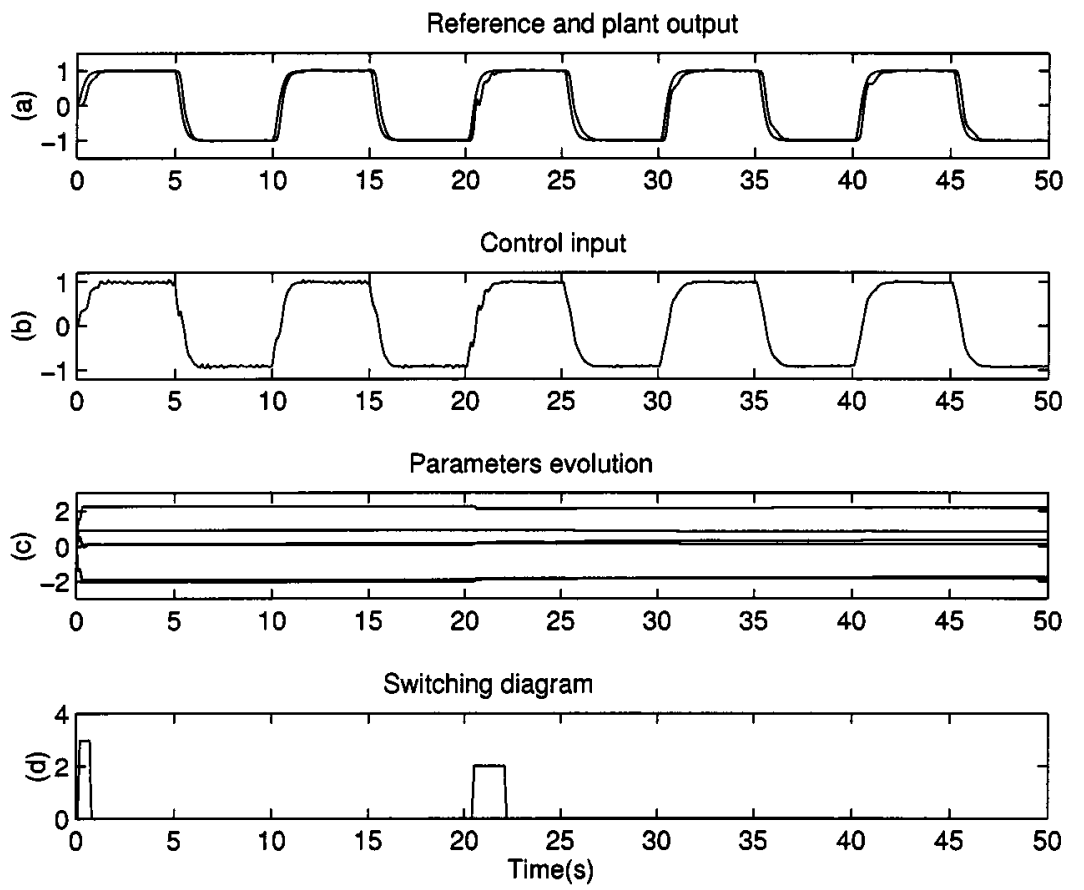

Fig. 14. Results of experiment $3(75 \% \rightarrow 25 \%$ in one stage at $19 \mathrm{~s})$.

\section{Experiment 3}

In order to show how the control system works, especially to emphasize the switching and tuning aspects, the second experiment will be carried out again passing from $75 \%$ load to $25 \%$ load in one stage. This experiment is particular because neither the initial model nor the final model belong to the set of fixed models used in the control scheme. The switching diagram of Fig. 14 shows that at the beginning of the experiment the full loaded model is chosen by the supervisor as the best model for the plant with $75 \%$ load (switching). Then the adaptive model initialized by the parameters of the full loaded model is chosen by the supervisor (from $1 \mathrm{~s}$ ) and remains as the best model for a period of about $20 \mathrm{~s}$ (tuning). Next, just after a load change on the third pulley (19s), the half loaded model is chosen rapidly as the best model for the plant with $25 \%$ load (switching) and then the parameters of the adaptive model are tuned by the adaptation algorithm (tuning). This experiment clearly shows the role 
of switching to the fixed models in augmenting the adaptation speed of the adaptive control system even when the true plant models do not correspond to the fixed models used in the control scheme.

\section{CONCLUSIONS}

An application of the multiple models adaptive control to a flexible transmission system has been presented. The robustness of the control system with respect to high-frequency uncertainties has been improved with the particular choice of the auxiliary poles in the pole placement technique.

A recently developed recursive adaptation algorithm has been used in the tuning phase of the multiple models scheme. It has been shown that the parameter adaptation in this algorithm is insensitive to the output disturbances and gives no parameter drift in the absence of excitation signal. The use of this algorithm in the multiple models adaptive control has the following advantages:

- It reduces the unwanted switching caused by the output disturbances.

- It gives a more precise model in the critical zone for robust control.

- The estimated parameters are not influenced by noise (asymptotically).

Thus, using this adaptation algorithm the performance of the control system is improved particularly in the tuning phase.

\section{REFERENCES}

[1] I. D. Landau, D. Rey, A. Karimi, A. Voda, and A. Franco, "A flexible transmission system as a benchmark for robust digital control," Eur. J. Contr., vol. 1, no. 2, 1995.

[2] N. W. Jones and D. J. N. Limebeer, "A digital $H_{\infty}$ controller for a flexible transmission system," Eur. J. Contr., vol. 1, no. 2, 1995.

[3] D. J. Walker, "Control of a flexible transmission-A discrete-time $H_{\infty}$ approach," Eur. J. Contr., vol. 1, no. 2, 1995.

[4] H. Kwakernaak, "Symmetries in control system design," in Trends in Control, A. Isidori, Ed. London, U.K.: Springer-Verlag, 1995.

[5] O. Kidron and O. Yaniv, "Robust control of uncertain resonant systems," Eur. J. Contr., vol. 1, no. 2, 1995.

[6] M. Nordin and P. Gutman, "Digital qft design for the benchmark problem," Eur. J. Contr., vol. 1, no. 2, 1995.

[7] A. Oustaloup, B. Mathieu, and P. Lanusse, "The crone control of resonant plants: Application to a flexible transmission," Eur. J. Contr., vol. 1, no. 2, 1995.

[8] C. Decker, A. Ehrlinger, P. Boucher, and D. Dumur, "Application of constrained receding horizon predictive control to a benchmark problem," Eur. J. Contr., vol. 1, no. 2, 1995.

[9] I. D. Landau, A. Karimi, A. Voda, and D. Rey, "Robust digital control of flexible transmission using the combined pole placement/sensitivity function shaping method," Eur. J. Contr., vol. 1, no. 2, 1995.

[10] I. D. Landau and A. Karimi, "Robust digital control using pole placement with sensitivity function shaping method," Int. J. Robust Nonlinear Contr., vol. 8, pp. 191-210, 1998.

[11] H. Hjalmarsson, S. Gunarsson, and M. Gevers, "Model free tuning of a robust regulator for a flexible transmission system," Eur. J. Contr., vol. 1, no. 2, 1995.
[12] M. M'Saad and I. Hejda, "Adaptive control of a flexible transmission system," Contr. Eng. Practice, vol. 2, no. 4, pp. 629-639, 1994.

[13] K. Narendra and Balakrishnan, "Adaptive control using multiple models," IEEE Trans. Automat. Contr., vol. 42, no. 2, pp. 171-187, Feb. 1997

[14] I. D. Landau and A. Karimi, "An output error recursive algorithm for unbiased identification in closed loop," Automatica, vol. 33, no. 5, pp. 933-938, May 1997.

[15] A. S. Morse, "Control using logic-based switching," in Trends in Control, A. Isidori, Ed. Heidelberg, Germany: Springer-Verlag, 1995.

[16] A. S. Morse, D. Q. Mayne, and G. C. Goodwin, "Application of hysteresis switching in parameter adaptive control," IEEE Trans. Automat. Contr., vol. 37, pp. 1343-1354, Sept. 1992.

[17] K. S. Narendra and J. Balakrishnan, "Improving response of adaptive control systems using multiple models and switching," IEEE Trans. Automat. Contr., vol. 39, pp. 1861-1866, Sept. 1994.

[18] A. Karimi and I. D. Landau, "Comparison of the closed-loop identification methods in terms of the bias distribution," Syst. Contr. Lett., no. 34, pp. 159-167, 1998.

[19] A. Karimi, "Conception des Régulateurs Numériques Robustes et Adaptatifs," Ph.D. dissertation, Institut National Polytechnique de Grenoble, LAG, Grenoble, France, 1997.

[20] , 1996.

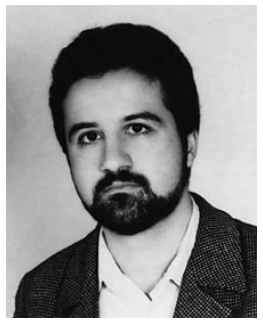

Alireza Karimi was born in Mashad, Iran, in 1964. He received the B.S. and M.S. degrees from Amir Kabir University (Teheran Polytechnic), Teheran, Iran, both in electrical engineering, in 1987 and 1990 , respectively, and the $\mathrm{Ph}$. D. degree in automatic control from National Polytechnic Institute, Grenoble, France, in 1997.

He was in charge of Research and Development Department in Iran Switch and Sapta companies from 1988 to 1993 . He is currently Associate Professor at Electrical Engineering Department of Sharif University of Technology. His research interests include closed-loop identification and adaptive and robust control.

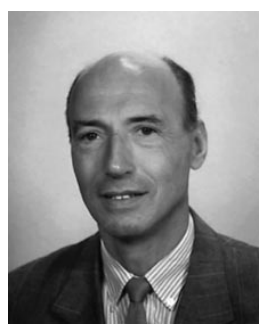

Ioan Doré Landau is Research Director at C.N.R.S. (National Centre for Scientific Research) and works at the Laboratoire d'Automatique de Grenoble (CNRS/INPG) of the National Polytechnic Institute of Grenoble. His research interests encompass theory and applications in system identification, adaptive control, robust digital control and nonlinear systems. He has authored and coauthored more than 200 papers on these subjects. He is the author of the books: Adaptive Control-The Model Reference Appoach (New York: Marcel Dekker, 1979) translated also in Chinese, System Identification and Control Design (Englewood Cliffs, NJ: Prentice-Hall, 1990), and coauthor (with M. Tomizuka) of the book Adaptive Control-Theory and Practice (Tokyo, Japan: Ohm, 1981, in Japanese). In 1997 he published the book Adaptive Control (New York: Springer-Verlag) coauthored with R. Lozano and M. M'Saad.

Dr. Landau received the Great Gold Medal at the Invention Exibition Vienna in 1968, the C.N.R.S. Silver Medal in 1982, the Best Review Paper Award (1981 to 1984) for his paper on adaptive control published in ASME Journal of Dynamical Systems Measurement and Control, and the prize Monpetit from the French Academy of Science. He was R. Springer Professor at the University of California, Berkeley, Department of Mechanical Engineering, in 1992. He was the the first President of the European Union Control Association (EUCA) from 1991 to 1993 and he is Editor in Chief of the European Journal of Control (a publication of the European Union Control Association). 